\title{
Microguidewire Looping to Traverse Stented Parent Arteries of Intracranial Aneurysms
}

\author{
Young Dae Cho, M.D., Jong Kook Rhim, M.D., ${ }^{1}$ Dong Hyun Yoo, M.D., ${ }^{1}$ Hyun-Seung Kang, M.D., Ph.D., ${ }^{2}$ \\ Jeong Eun Kim, M.D., Ph.D., ${ }^{2}$ Moon Hee Han, M.D., Ph.D., \\ Department of Radiology, Department of Neurosurgery, Seoul National University Hospital, Seoul National University College of Medicine, \\ Seoul, Korea
}

Objective : Stents are widely used in coil embolization of intracranial aneurysms, but on occasion, a microcatheter must traverse a stented segment of artery (so-called trans-cell technique) to select an aneurysm, or double stenting may necessary. In such situations, microguidewire passage and microcatheter delivery through a tortuous stented parent artery may pose a technical challenge. Described herein is a microguidewire looping technique to facilitate endovascular navigation in these circumstances.

Methods : To apply this technique, the microguidewire tip is looped before entering the stented parent artery and then advanced distally past the stented segment, with the loop intact. Rounding of the tip prevents interference from stent struts during passage. A microcatheter is subsequently passed into the stented artery for positioning near the neck of aneurysm, with microguidewire assistance. The aneurysm is then selected, steering the microcatheter tip (via inner microguidewire) into the dome.

Results : This technique proved successful during coil embolization of nine saccular intracranial aneurysms (internal carotid artery [ICA], 6; middle cerebral artery, 2; basilar tip, 1), performing eight trans-cell deliveries and one additional stenting. Selective endovascular embolization was enabled in all patients, resulting in excellent clinical and radiologic outcomes, with no morbidity or mortality directly attributable to microguidewire looping.

Conclusion : Microguidewire looping is a reasonable alternative if passage through a stented artery is not feasible by traditional means, especially at paraclinoid ICA sites.

Key Words : Aneurysm · Coil · Embolization · Microguidewire · Stent.

\section{INTRODUCTION}

Endovascular treatment of intracranial aneurysms has flourished in the aftermath of two pivotal studies : the International Subarachnoid Aneurysm Trial (ISAT) and the International Study of Unruptured Intracranial Aneurysms
(ISUIA). Device improvements and advances in coiling techniques have now enabled treatment of lesions with complex configurations $^{3,18)}$. Stenting (and various modifications thereof) has contributed greatly in this context, broadening the scope of endovascular treatment substantially, ${ }^{5,710,12,14-19,21)}$. When inserting coil under stent protection, a jailed catheter

- Received : July 19, 2016 •Revised : December 8, 2016 •Accepted : December 23, 2016

- Address for reprints : Hyun-Seung Kang, M.D., Ph.D.

Department of Neurosurgery, Seoul National University Hospital, Seoul National University College of Medicine, 101 Daehak-ro, Jongno-gu, Seoul 03080, Korea Tel : +82-822-2072-1351, Fax : +82-822-744-8459, E-mail : ydofmh@gmail.com

This is an Open Access article distributed under the terms of the Creative Commons Attribution Non-Commercial License (http://creativecommons.org/licenses/by-nc/4.0) which permits unrestricted non-commercial use, distribution, and reproduction in any medium, provided the original work is properly cited. 
technique is generally applied first-line. However, should jailed technique fail or fall short in terms of coil compaction, passing a microcatheter through stent struts (so-called transcell technique) may be required to satisfactorily complete coil embolization. Additional stenting may also be needed in specific conditions, such as dissecting, fusiform, and blood-blister aneurysms, or as bail-outs solutions. Still, passage of both microguidewire and microcatheter through a stented parent artery is not easy, given the potential interference by stent struts, and it is particularly difficult in the tortuous carotid siphon. Recently, we devised a microguidewire looping method to easily traverse a stented parent artery. Feasibility and safety of this approach were explored in a limited group of patients, whose angiographic and clinical outcomes are detailed herein.

\section{MATERIALS AND METHODS}

\section{Study population}

Between January, 2013 and April, 2015, 982 aneurysms in 840 patients were subjected to endovascular coil embolization, including 249 instances (25.4\%) of stent usage. Of these, 138 (55.4\%) were amenable to jailed technique only, 35 (14.1\%) required stenting upon completion of coil insertion (such as bail-out stenting), and 76 (30.5\%) called for trans-cell technique ( 68 by traditional means). In 10 patients, more than two stents were deployed.

Microguidewire looping was applied in nine subjects (female, 8 ; male, 1 ; mean age, $61.9 \pm 5.9$ years), largely due to failed or difficult traditional microguidewire advancement. One patient required additional stenting. All aneurysms were unruptured and broad necked, with depth-to-neck ratios $<1.5$ (mean, $0.9 \pm 0.2$; range, $0.5-1.2)$ and small diameters $(<10 \mathrm{~mm})$. Therapeutic decisions relied on multidisciplinary deliberation by both neurosurgeons and nonsurgical neurointerventionists. Once the need for treatment and related risks were adequately explained, all patients granted informed consent. This study was approved by the Institutional Review Board of our institution. Clinical and radiographic features of the cohort are shown in Table 1.

\section{Therapeutic strategies}

Endovascular access by microguidewire looping was achieved step-wise as follows : 1) loop created in microguide-

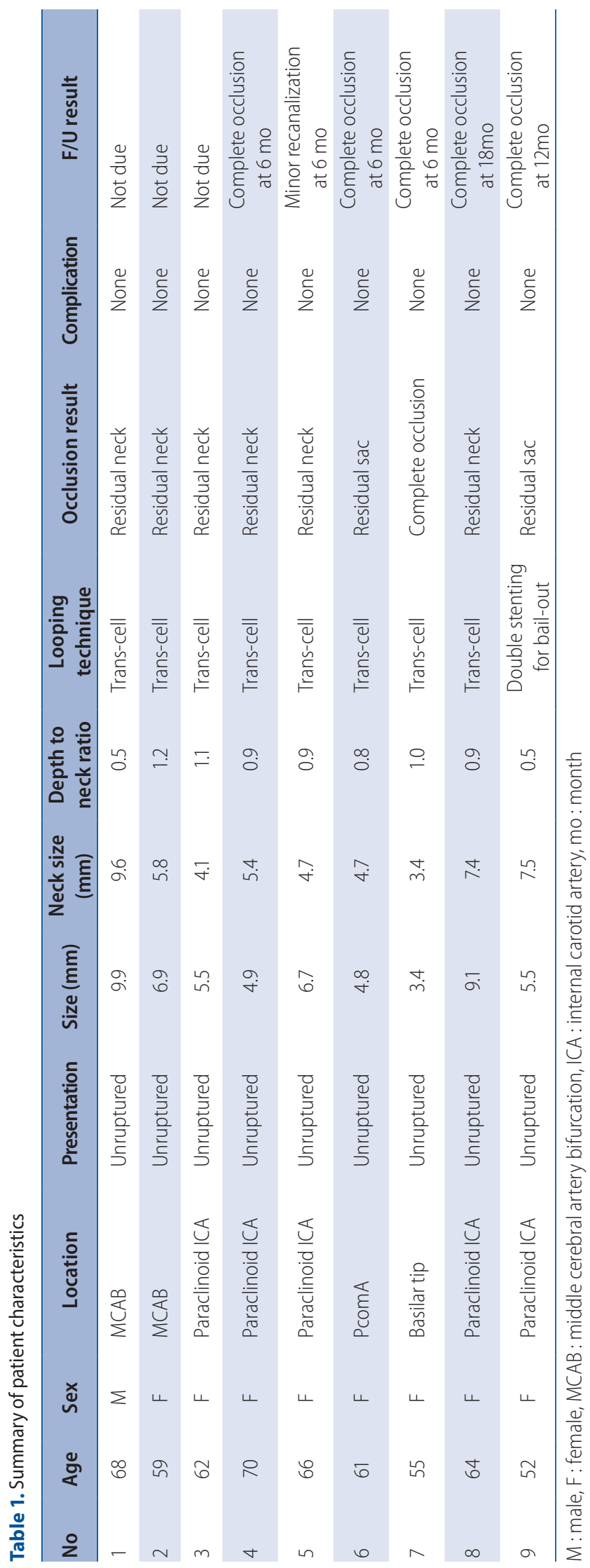


wire tip; 2) advancement of looped microguidewire distally, across stented segment; 3 ) passage of microcatheter (microguidewire-assisted) close to aneurysm; 4) trans-cell navigation of microcatheter tip (via inner microguidewire) into dome of aneurysm. Schematic representation is depicted in Fig. 1.

The first step in this maneuver is looping the microguidewire tip. There are two ways to loop a distal microguidewire tip. First, the tip of microguidewire should be shaped with very small and tight angle. When this shaped microguidewire passes the petrocavernous internal carotid artery (ICA) (Fig. 2A, B) or the proximal portion of a stent (Fig. 2C, D), a loop can be easily formed. Should this maneuver fail, entry into a small arterial branch, such as the meningohypophysial trunk, may be productive (Fig. 2E-G). Once created, the loop should be advanced distally, through the stented segment of parent artery. A rotational change in loop orientation may help surmount any stent interference. After positioning the microguidewire as needed, the microcatheter should follow. The aneurysm selection is achieved using inner microguidewire torque to navigate the microcatheter through the stent struts.

In advancing a microguidewire, the tip usually makes contact with arterial wall. Struts incompletely in apposition are

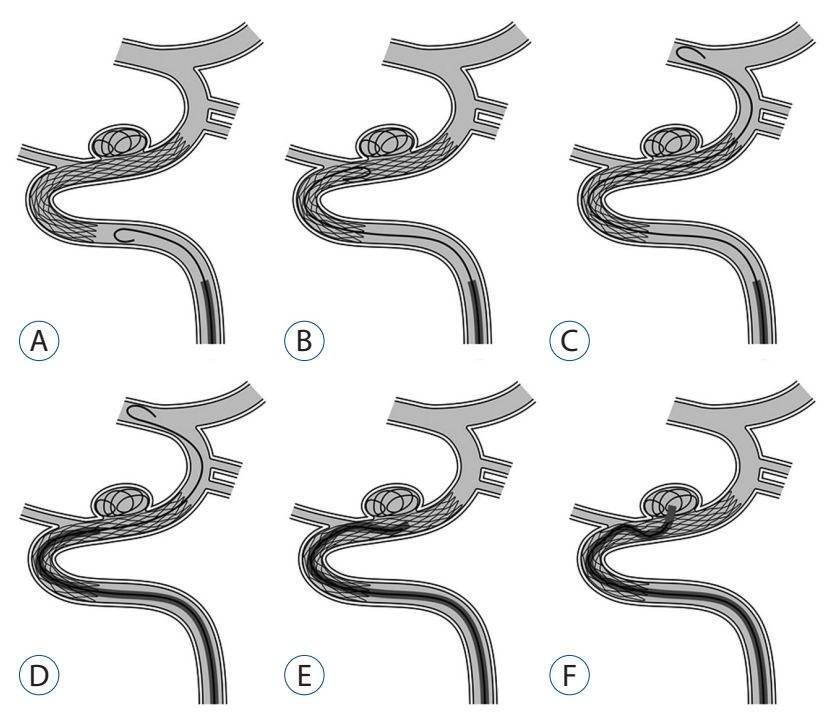

Fig. 1. Microguidewire looping technique (to navigate stented parent artery). A : Microguidewire loop shaped before or at proximal stented segment after proper configuring of wire tip. B and C : Looped microguidewire tip advanced to distal parent artery, passing through stented segment. D : Microcatheter follows (microguidewire-assisted), advanced near neck of aneurysm. E : Microguidewire withdrawal to tip of microcatheter. F: Microcatheter eased into sac of aneurysm under microguidewire torque. thus likely to retard navigation, especially in lesions with tortuous parent arteries. Ordinarily, stent struts must be avoided to prevent microguidewire entanglement, but the rounded contour of a looped microguidewire facilitates passage, minimizing this risk. Nevertheless, this looping technique is only warranted if traditional methods are ineffective.

\section{Endovascular procedure}

The configuration of each aneurysm and its arterial grid were delineated using Allura Clarity (Philips Medical System, Best, The Netherlands) and Innova IGS 630 (General Electric, Wauwatosa, WI, USA) biplane systems, providing high-resolution 3D rotational angiography. The size of an aneurysm was defined as its broadest aspect in 3D configuration. Neck size and depth-to-neck ratio were measured on a working projection of digital subtraction angiography.

All procedures were performed under general anesthesia, administering antiplatelet medication in advance. In accord
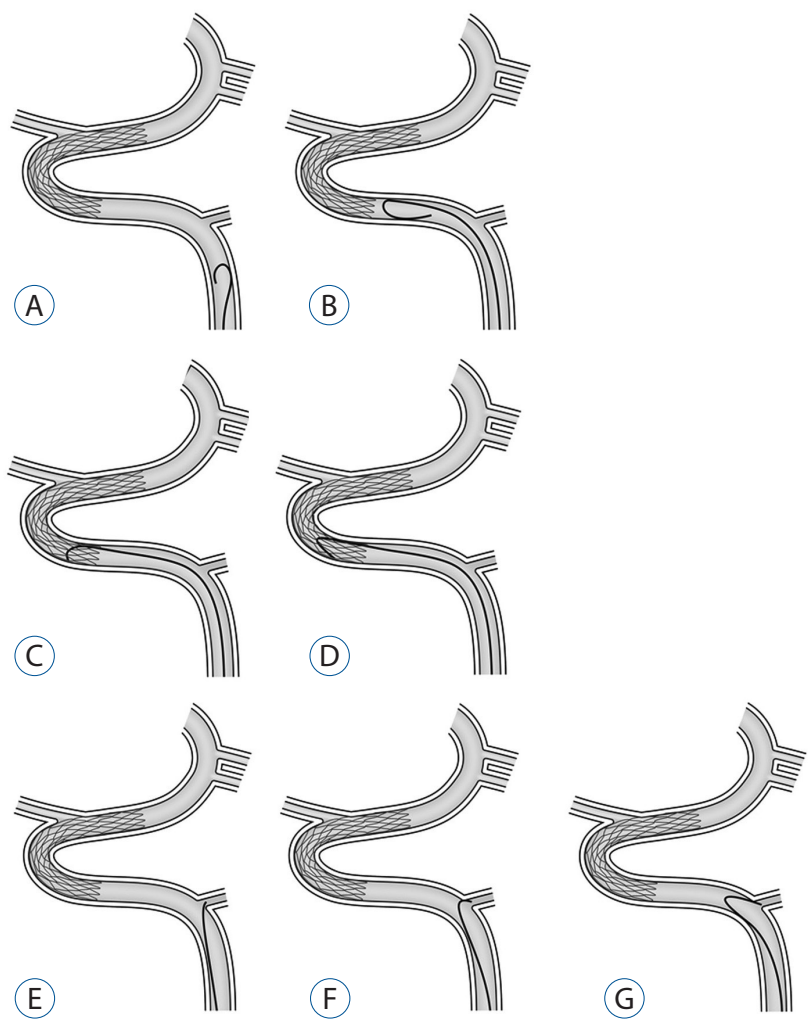

Fig. 2. Configuring microguidewire loop. $A$ and $B$ : Acute-angled tip required. $C$ and $D:$ Use of proximal stent struts to loop microguidewire tip. E, F and G : Looping via small branch vessel (eg, meningohypophyseal trunk). 
with institutional protocol, patients were given dual antiplatelet pre-medication (clopidogrel and aspirin). In patients responding poorly to clopidogrel, based on VerifyNow P2Y12 assay (Accriva Diagnostics, San Diego, CA, USA) ${ }^{11}$, cilostazol was added. A bolus of heparin (3000 IU) was given after femoral arterial sheath placement, with hourly bolus doses (1000 IU each) thereafter. Activated clotting time was also monitored hourly. Maintenance antiplatelet medication was then advised, using dual agents for at least 3 months after the procedure and a single agent for at least 1 year.

\section{Angiographic outcome and follow-up}

Immediate angiographic results after coil embolization were interpreted by two experienced neurointerventionists (YDC and HSK), applying the 3-point Raymond scale as follows : complete occlusion (no residual filling of aneurysms by contrast medium), residual neck (limited residual contrast at base of aneurysm), or residual aneurysm (any opacification of the sac) ${ }^{20)}$.

Clinical outcomes were scored by Glasgow outcome scale (GOS) throughout the hospitalization period and at the last available clinical follow-up. In all patients, magnetic resonance angiography (MRA) with 3D reconstruction and/or plain radiography was recommended at 6, 12, 24, and 36 months after coil embolization. Conventional angiography was advised if aneurysmal recanalization was suspected by noninvasive evaluation, such as MRA or plain radiography, in order to decide if further treatment was necessary.

Anatomic follow-up results were also categorized by Raymond scale as follows : complete occlusion, minor recanalization, or major recanalization. Repeat embolization was advocated for patients showing major recanalization.

\section{RESULTS}

Our method of microguidewire looping, in conjunction with trans-cell technique $(\mathrm{n}=8)$ and in one additional bail-out stenting (due to coil protrusion), proved successful in nine subjects with saccular aneurysms of ICA $(n=6)$, middle cerebral artery (MCA ; $n=2)$, and basilar tip $(n=1)$. The Enterpri$\mathrm{se}^{\mathrm{TM}}$ stent (Codman Neuro, Raynham, MA, USA) was primarily used ( $\mathrm{n}=7$ ), followed by LVIS ${ }^{\mathrm{TM}}$ (MicroVention Inc, Tustin, CA, USA) and Neuroform ${ }^{\text {TM }}$ (Stryker Neurovascular, Fre- mont, CA, USA) stents (one patient each). Selective stent-assisted coil embolization was thereby facilitated, resulting in excellent outcomes for all patients.

Immediately following coil embolization, seven lesions were successfully occluded (complete occlusion, 1; neck remnant, 6), and two retained small residual sacs. There were no adverse events during procedures, and no morbidity or mortality resulted as a direct consequence of microguidewire looping. All patients were neurologically intact (GOS 5) upon discharge.

Six patients (excluding three treated recently) underwent follow-up evaluations at $>6$ months, including MRA or conventional angiography. In five instances, complete aneurysmal occlusion was sustained, and one showed minor recanalization. None of the patients experienced delayed complications (ie, thromboembolic infarction or hemorrhage).

\section{Illustrative case 1}

A 62-year-old woman was admitted for endovascular treatment of an unruptured distal ICA aneurysm (maximum size, $4.8 \mathrm{~mm}$; dome-to-neck ratio, 0.8 ). Her family history of subarachnoid hemorrhage prompted treatment. A 6-Fr guiding catheter was passed into cervical ICA. Given the wide neck of the aneurysm, coil insertion by jailed technique was performed first-line, after stent deployment. Although the frame coil was established and properly configured, unsuitable microcatheter orientation prevented additional coiling. The jailed microcatheter was then withdrawn to proximal stent position, and trans-cell technique was applied. When the stented segment could not be negotiated by traditional means, passage was ultimately achieved through microguidewire looping. A microcatheter was finally passed to point near the neck of aneurysm and was directed into the sac using inner microguidewire torque. Insertion of additional coil was thus enabled, resulting in minimal residual filling of an otherwise occluded sac (Fig. 3). The patient was discharged the following day, free of complications. At the 6-month follow-up visit, MRA confirmed complete occlusion of the aneurysm.

\section{Illustrative case 2}

This 52-year-old female was admitted for endovascular treatment of a left-sided aneurysm of paraclinoid ICA. By conventional angiography, a very wide neck (depth-to-neck ratio, 0.5 ) was noted, so coil embolization was performed via jailed microcatheter under stent protection. Due to a loop of 

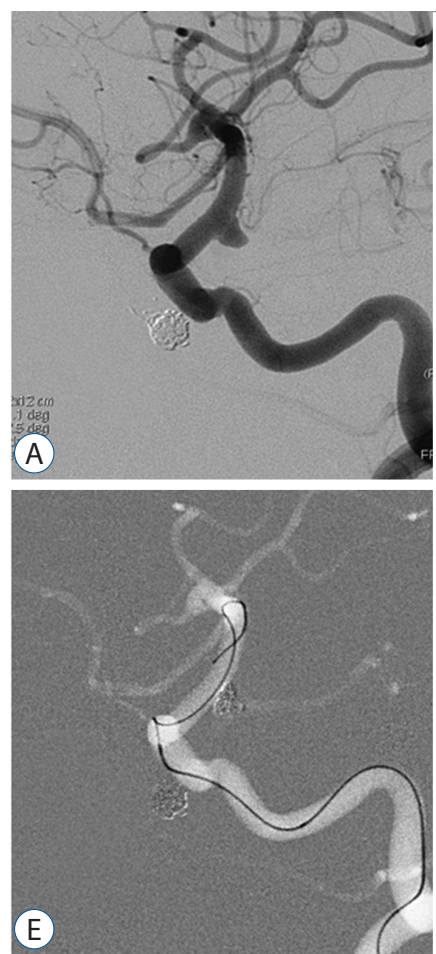
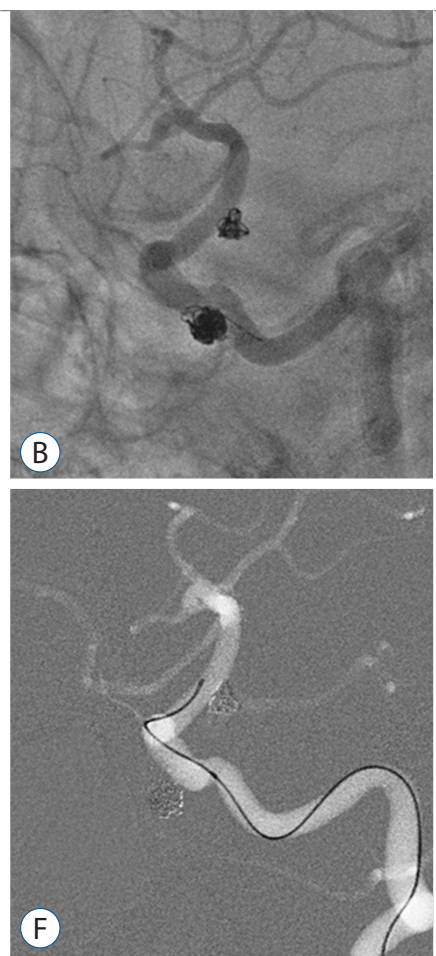
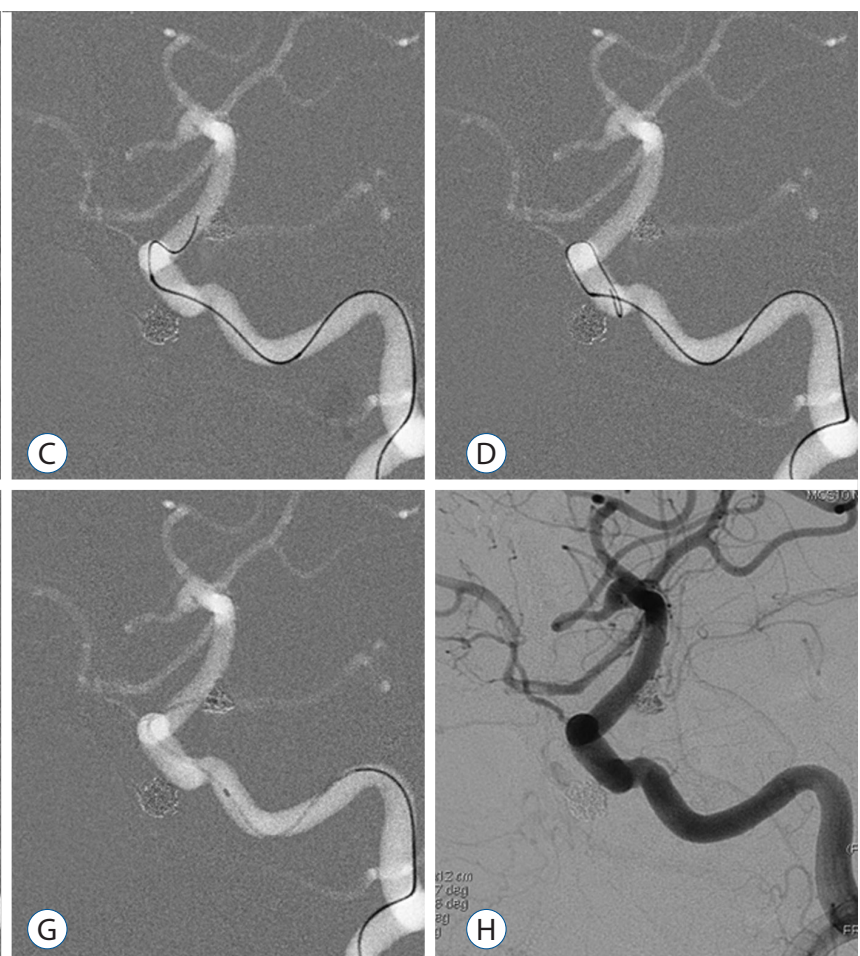

Fig. 3. A : Angiographic image of distal ICA aneurysm. B : Frame coil formed and properly configuration by jailed method, after stent deployment. C : Unsuitably oriented jailed microcatheter withdrawn to proximal stent position, but traditional means fail to advance microguidewire. $D$ : Microguidewire loop formed before proximal stent reached. $\mathrm{E}$ : Looping technique enables microguidewire advancement. F : Passage of microcatheter to point near aneurysm. G : Aneurysm selected via inner microguidewire torque. $\mathrm{H}$ : Additional coil insertion occludes aneurysm, with minimal residual filling. ICA : internal carotid artery.

filler coil protruding into parent artery, additional stenting was required. Use of our microguidewire looping technique allowed passage of microdevices for stent delivery to MCA, and an additional stent was deployed. The aneurysm was satisfactorily occluded, without complication, and was unchanged 1 year later in follow-up angiography (Fig. 4).

\section{DISCUSSION}

Recent advancements in design and delivery of stents have ensured their use with ease and confidence ${ }^{4)}$. Self-expanding stents that promote higher packing density, flow diversion, and endothelization at necks of aneurysms are gaining in popularity ${ }^{2,13)}$. The actual course of a parent artery may be altered as well ${ }^{6}$. Even stent devices with low wall coverage divert flow in small aneurysms ${ }^{15}$, so enhanced wall coverage through smaller cell size may augment this effect, promoting a very high rate of occlusion. These effects favorably impact hemodynamic conditions near an aneurysm and help reduce recan- alization rates.

In practice, a variety of stents are used for coil embolization of intracranial aneurysms, including open- and closed-cell designs, braided and laser-cut styles, and more. In general, a first-line approach for stent-assisted coil embolization is jailed technique, which alone is adequate for treating many lesions. In our series, use of jailed technique to complete coil embolization was applied in $55.4 \%$ of aneurysms involving stents. However, trans-cell technique is sometimes required if jailed technique should fail or fall short in the coiling process. It is well-known that stenting at sharply angled segments of parent arteries are troubled by incomplete apposition. This phenomenon is more pronounced in a closed-cell design, often manifested as kinking or folding ${ }^{8)}$. Heller et al. .) $^{9}$ reported doublebarrel entanglement of intracranial stents resulting from undetected incomplete apposition of stent to vessel wall. In such circumstances, the technical demands of trans-cell technique or further stenting are high, especially in aneurysms near tortuous parent arteries (eg, paraclinoid ICA aneurysms). Even if apposition is complete, stent struts may still interfere 

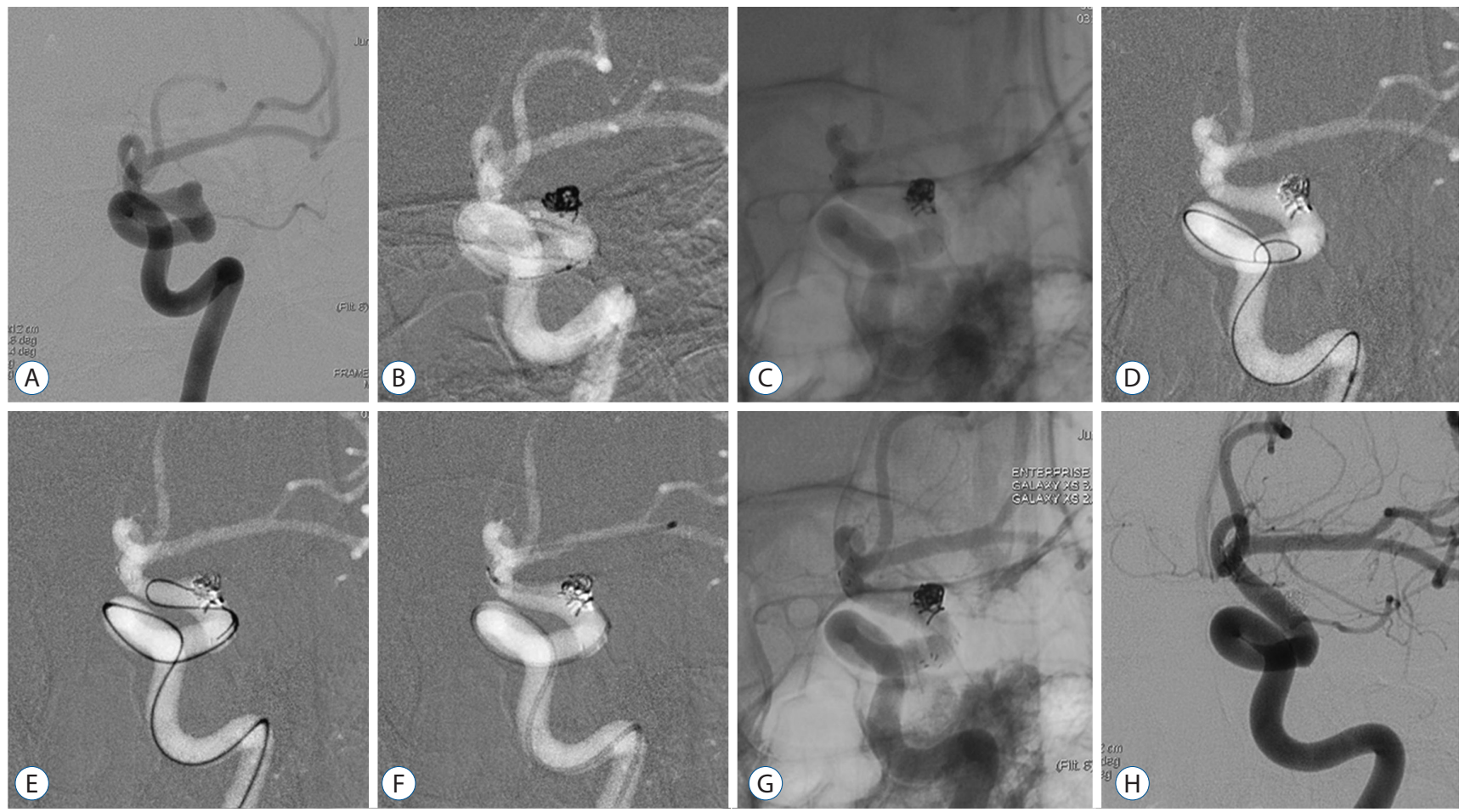

Fig. 4. A : Angiographic image of wide-necked paraclinoid ICA aneurysm. B : Frame and filler coils inserted by jailed method, after stent deployment. C : Last coil protrudes into parent artery, requiring additional stenting for stabilization. D, E and F : Microcatheter for stent delivery advanced into MCA via looped microguidewire. G : Additional stenting deployed and aneurysm satisfactorily occluded. $\mathrm{H}$ : Complete occlusion of aneurysm 1 year later in follow-up angiographic image. ICA : internal carotid artery, MCA : middle cerebral artery.

Table 2. Traditional maneuvers ( $n=68)$ vs. microguidewire looping $(n=8)$ during trans-cell coil embolization $(n=76)$, shown by location (one double stenting excluded).

\begin{tabular}{lcc}
\hline Location $(\mathbf{n}=\mathbf{7 6})$ & Traditional maneuver $(\mathbf{n}=\mathbf{6 8})$ & Microguidewire looping $(\mathbf{n}=\mathbf{8})$ \\
\hline ICA including PcomA $(n=25)$ & $20(80)$ & $5(20)$ \\
MCA $(n=14)$ & $12(86)$ & $2(14)$ \\
ACA including AcomA $(n=18)$ & $18(100)$ & $0(0)$ \\
Posterior circulation $(n=19)$ & $18(95)$ & $1(5)$ \\
\hline
\end{tabular}

Values are presented as number (\%). ICA : internal carotid artery, PcomA : posterior communicating artery, MCA : middle cerebral artery, ACA : anterior cerebral artery, AcomA : anterior communicating artery

with the passage of microdevices. Microguidewire looping is an effective alternative method for negotiating stented vessels if a traditional approach has failed. Rounding of the tip minimizes its capacity to snare an incompletely apposed strut.

In this series, looping was most often utilized for paraclinoid ICA aneurysms, which are more tortuous by nature. The obstacles to traditional wire torque were thus intensified. There was also a greater likelihood of incomplete stent apposition at this location. Hence, our novel technique may be of less value in MCA or basilar tip aneurysms, where parent ar- teries are relatively straight in course. Likewise, microguidewire looping is not appropriate for anterior cerebral or anterior communicating arteries, because the Al segment is virtually inaccessible this way (Table 2). Furthermore, stent migration may result, particularly if the stent is a closed-cell design, although this was not a problem here. In ruptured aneurysms, migration of clot on stent might occur. In other settings, this approach may facilitate microdevice delivery into true distal lumen of a direct carotid-cavernous fistula or an acute dissection. According to Benndorf et al. ${ }^{1)}$, microgu- 
idewire looping enabled breach of an occluded inferior petrosal sinus in the context of cavernous sinus dural arteriovenous fistula.

We do not advocate microguidewire looping as a first-line technique for negotiating stented arteries. If feasible by traditional means, this looping method is not needed. Although its use in disadvantaged situations may certainly be justified, the efficacy and safety of this approach must be confirmed in larger study populations.

\section{CONCLUSION}

Looping of a distal microguidewire tip is a viable option for accessing the distal reaches of stented parent arteries during trans-cell technique or if added stenting is required, but only if traditional methods have failed or are untenable. This technique is particularly suitable for paraclinoid ICA aneurysms.

\section{References}

1. Benndorf G, Bender A, Lehmann R, Lanksch W : Transvenous occlusion of dural cavernous sinus fistulas through the thrombosed inferior petrosal sinus : report of four cases and review of the literature. Surg Neurol $54:$ 42-54, 2000

2. Chalouhi N, Jabbour P, Singhal S, Drueding R, Starke RM, Dalyai RT, et al. : Stent-assisted coiling of intracranial aneurysms : predictors of complications, recanalization, and outcome in 508 cases. Stroke 44 : 1348-1353, 2013

3. Cho YD, Kang HS, Kim JE, Son YJ, Lee JY, Lee SJ, et al. : Microcatheter looping technique for coil embolization of complex configuration middle cerebral artery aneurysms. Neurosurgery 71 : 1185-1191; discussion 1191, 2012

4. Cho YD, Lee JY, Seo JH, Lee SJ, Kang HS, Kim JE, et al. : Does stent implantation improve the result of repeat embolization in recanalized aneurysms? Neurosurgery 71(2 Suppl Operative) : ons253-ons259; discussion ons259, 2012

5. Cho YD, Park SW, Lee JY, Lee JY, Seo JH, Kang HS, et al. : Nonoverlapping Y-configuration stenting technique with dual closed-cell stents in wide-neck basilar tip. Neurosurgery 70(2 Suppl Operative) : 244249, 2012

6. Cho WS, Kang HS, Kim JE, Kwon OK, Oh CW, Cho YD, et al. : Angle change of the parent arteries after stent-assisted coil embolization of wide-necked intracranial bifurcation aneurysms. Clin Radiol 69 : e63e70, 2014

7. Gory B, Klisch J, Bonafé A, Mounayer C, Beaujeux R, Moret J, et al. : Solitaire $A B$ stent-assisted coiling of wide-necked intracranial aneurysms : short-term results from a prospective, consecutive, European multicentric study. Neuroradiology 55 : 1373-1378, 2013

8. Heller RS, Malek AM : Parent vessel size and curvature strongly influence risk of incomplete stent apposition in enterprise intracranial aneurysm stent coiling. AJNR Am J Neuroradiol 32 : 1714-1720, 2011

9. Heller RS, Safain M, Malek AM : Double-barrel entanglement of intracranial Enterprise stents resulting from undetected incomplete stent apposition. J Neurointerv Surg 4 : e38, 2012

10. Juszkat R, Nowak S, Smól S, Kociemba W, Blok T, Zarzecka A : Leo stent for endovascular treatment of broad-necked and fusiform intracranial aneurysms. Interv Neuroradiol 13 : 255-269, 2007

11. Kang HS, Kwon BJ, Kim JE, Han MH : Preinterventional clopidogrel response variability for coil embolization of intracranial aneurysms : clinical implications. AJNR Am J Neuroradiol 31 : 1206-1210, 2010

12. Kulcsár Z, Göricke SL, Gizewski ER, Schlamann M, Sure U, Sandalcioglu IE, et al. : Neuroform stent-assisted treatment of intracranial aneurysms : long-term follow-up study of aneurysm recurrence and in-stent stenosis rates. Neuroradiology 55 : 459-465, 2013

13. Lawson MF, Newman WC, Chi YY, Mocco JD, Hoh BL : Stent-associated flow remodeling causes further occlusion of incompletely coiled aneurysms. Neurosurgery 69 : 598-603, 2011

14. Lee SJ, Cho YD, Kang HS, Kim JE, Han MH : Coil embolization using the self-expandable closed-cell stent for intracranial saccular aneurysm : a single-center experience of 289 consecutive aneurysms. Clin Radiol $68: 256-263,2013$

15. Liu H, Choe J, Jung SC, Song Y, Yang KH, Park KJ, et al. : Does a lowwall coverage stent have a flow diverting effect in small aneurysms? Neurointervention $10: 89-93,2015$

16. Lubicz B : Linear stent-assisted coiling : another way to treat very widenecked intracranial aneurysms. Neuroradiology 53 : 457-459, 2011

17. Park KY, Kim BM, Kim DJ : Comparison between balloon-assisted and stent-assisted technique for treatment of unruptured internal carotid artery aneurysms. Neurointervention 11 : 99-104, 2016

18. Park W, Song Y, Park KJ, Koo HW, Yang K, Suh DC : Hemodynamic characteristics regarding recanalization of completely coiled aneurysms : Computational fluid dynamic analysis using virtual models comparison. Neurointervention 11 : 30-36, 2016

19. Piotin M, Blanc R, Spelle L, Mounayer C, Piantino R, Schmidt PJ, et al. : Stent-assisted coiling of intracranial aneurysms : clinical and angiographic results in 216 consecutive aneurysms. Stroke 41 : 110-115, 2010

20. Roy D, Milot G, Raymond J : Endovascular treatment of unruptured aneurysms. Stroke 32 : 1998-2004, 2001

21. Yashar P, Kan PT, Levy El : Horizontal deployment of an intracranial stent via an antegrade approach for coil embolization of a basilar apex aneurysm : technical note. J Neurointerv Surg 3 : 355-357, 2011 